\title{
Comunicação
}

[Communication]

\section{Helmintos e protozoários em fezes de javalis (Sus scrofa scrofa) criados em cativeiro}

\author{
[Helminths and protozoa in wild boars (Sus scrofa scrofa) feces raised in captivity]
}

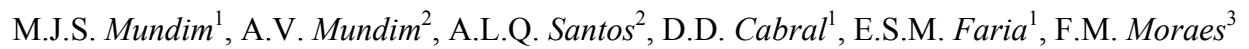 \\ ${ }^{1}$ Instituto de Ciências Biomédicas - Universidade Federal de Uberlândia \\ Campus Umuarama \\ Av. Para, 1720 \\ 38400-902 - Uberlândia, MG \\ ${ }^{2}$ Faculdade de Medicina Veterinária - UFU - Uberlândia \\ ${ }^{3}$ Acadêmico de Medicina Veterinária - UFU - Uberlândia
}

O javali é um suídeo silvestre nativo do continente africano, cuja carne é apreciada pelo homem, graças à sua qualidade, baixos teores de gordura e colesterol, poucas calorias e alto teor protéico. Quando comparada à de bovino, apresenta $85 \%$ menos calorias e $31 \%$ mais proteínas, despertando o interesse dos nutricionistas e consumidores que exigem cada vez mais uma dieta balanceada (Antunes, 2001).

Os javalis são acometidos por diversas parasitoses e conseqüentemente podem atuar como potenciais reservatórios para suínos domésticos e outras espécies animais. Embora estudos anteriores tenham sido realizados para identificar e determinar a prevalência de helmintos e protozoários na espécie, via encontro das formas adultas nas vísceras dos animais (Humbert e Henry, 1989; Eslami e FarsadHamdi, 1992; Gomes et al., 2002), pesquisas das parasitoses de javalis em cativeiro são raras.

O objetivo deste estudo foi identificar e determinar a freqüência dos endoparasitas em amostras de fezes de javalis em um criatório para fins comerciais localizado na região do Triângulo Mineiro.

Amostras de fezes de 79 javalis (40 machos e 39 fêmeas), em diferentes idades, foram coletadas para pesquisa de ovos, larvas, cistos e oocistos de parasitas. Os animais foram agrupados de acordo com a faixa etária em 57 jovens ( $\leq 12$ meses) e 22 adultos ( $>12$ meses). As amostras, coletadas diretamente da ampola retal e acondicionadas em sacos plásticos sem conservante, foram processadas pelo método de centrífugo-flutuação em solução de sulfato de zinco a 33\% (Faust et al., 1939) e método de sedimentação espontânea (Lutz, 1919). As amostras positivas para oocistos de coccídios em um dos métodos acima relacionados foram colocadas para esporular em solução de bicromato de potássio a $2,5 \%$ por um período de uma a quatro semanas, em temperatura ambiente. Após a esporulação, as amostras foram processadas pelo método de centrífugo-flutuação em solução de açúcar (Sheather, 1923). Os oocistos foram mensurados e a diferenciação das espécies foi feita segundo os critérios morfométricos adotados por Rodriguez e Herrera (1971).

Para verificar o efeito das faixas etárias sobre a freqüência das diferentes espécies de endoparasitas, utilizou-se o teste de hipóteses para diferença de proporção, com $5 \%$ de significância, segundo Triola (1999).

Setenta e sete amostras $(97,5 \%)$ foram positivas para endoparasitas; $72(91,1 \%)$ para helmintos e $61(77,2 \%)$ para protozoários. Dezesseis $(20,2 \%)$ amostras apresentaram somente helmintos, cinco

Recebido para publicação em 26 de junho de 2003

Recebido para publicação, após modificações, em 28 de novembro de 2003

E-mail: avmundim@demea.ufu.br 
$(6,3 \%)$ somente protozoários e $56 \quad(70,9 \%)$ infecção concomitante por protozoários e helmintos.

Os helmintos e protozoários identificados nas amostras fecais estudadas são comuns em fezes de suínos domésticos e silvestres. Os gêneros e espécies dos parasitas identificados nos animais de acordo com a faixa etária estão listados na Tab. 1

Tabela 1. Freqüência de ovos, larvas e cistos de endoparasitas identificados em 79 amostras fecais de javalis (Sus scrofa scrofa), criados em cativeiro de acordo com a faixa etária

\begin{tabular}{|c|c|c|c|c|c|c|}
\hline \multirow{2}{*}{ Endoparasita } & \multicolumn{2}{|c|}{$\leq 12$ meses $(n=57)$} & \multicolumn{2}{|c|}{$>12$ meses $(n=22)$} & \multicolumn{2}{|c|}{ Total $(n=79)$} \\
\hline & Positivo & $\%$ & Positivo & $\%$ & Positivo & $\%$ \\
\hline Estrongilídeos & 40 & 70,1 & 16 & 72,7 & 56 & 70,9 \\
\hline Ascaris suum & 23 & 40,3 & 14 & 63,6 & 37 & 46,9 \\
\hline Trichuris suis & 17 & 29,8 & 6 & 27,3 & 23 & 29,1 \\
\hline Metastrongylus spp. & 6 & 10,5 & 4 & 18,2 & 10 & 12,6 \\
\hline Strongyloides ransomi & 2 & 3,5 & 1 & 4,5 & 3 & 3,8 \\
\hline Balantidium coli & 22 & 38,5 & 8 & 36,4 & 30 & 38,0 \\
\hline Entamoeba spp. & 11 & $19,3^{*}$ & 1 & 4,5 & 12 & 15,2 \\
\hline Giardia spp. & 1 & 1,7 & - & - & 1 & 1,3 \\
\hline Blastocystis sp. & 8 & 14,0 & 2 & 9.0 & 10 & 12,6 \\
\hline
\end{tabular}

As pesquisas de identificação de helmintos que acometem os suídeos silvestres baseiam-se no encontro das formas adultas nas vísceras dos animais abatidos (Eslami e Farsad-Hamdi, 1992; Gomes et al., 2002).

Nove $\quad(11,4 \%) \quad$ animais apresentaram monoparasitismo, $21(26,5 \%)$ biparasitismo e 47 $(59,4 \%)$ poliparasitismo.

A freqüência de estrongilídeos, metastrongilídeos, Ascaris suum e Trichuris suis foram consideradas elevadas. Estudos têm identificado estes helmintos em suídeos silvestres e domésticos. Os metastrongilídeos são helmintos freqüentes em javalis (Humbert e Henry, 1989; Eslami e Farsad-Hamdi, 1992). No Brasil, Muller et al. (2001) detectaram o parasita em javalis procedentes de um criatório semiintensivo no Rio Grande do Sul. Os ascarídeos são também muito freqüentes e seus ovos apresentam longo período de resistência no meio ambiente. Isto deve ter facilitado a contaminação dos animais e contribuiu para a elevada freqüência detectada. Os animais jovens foram os mais acometidos, o que é comum em infecções por ascarídeos. A freqüência do Strongyloides ransomi foi baixa.

Animais com até 12 meses foram os mais acometidos para Entamoeba spp. em comparação com os acima de 12 meses $(\mathrm{P}<0,05)$, resultados condizentes com os de Pakandl (1994). Não foi possível realizar o cultivo da Entamoeba spp. para identificação da espécie.

Deve-se ressaltar a detecção das formas vacuolares do Blastocystis sp. nas fezes dos animais. Estudos anteriores detectaram este agente em fezes de suídeos domésticos e selvagens Sus scrofa (Belova, 1995). Atualmente, Blastocystis sp. tem sido muito estudado, contudo pouco se conhece sobre vários aspectos desse agente (Boreham e Stenzel, 1993), especialmente em animais silvestres.

A freqüência encontrada para Giardia spp. foi baixa, semelhante aos resultados de Atwill et al. (1997). Esses autores relatam que suídeos silvestres podem liberar cistos de Giardia spp. nas fezes, podendo servir como fonte de infecção, contaminando as águas de superfície. Esse protozoário apresenta importância em saúde pública. A freqüência de Balantidium coli foi considerada alta, concordando com os achados de Nakauchi (1999). Essa freqüência em suídeos silvestres é esperada pelo fato dos suínos serem considerados hospedeiros naturais desses protozoários na natureza, albergando o ciliado na maioria dos casos. 
Quarenta e sete amostras (59,5\%) apresentaram oocistos de coccídios, sendo 41 somente oocistos de Eimeria spp., quatro somente de Isospora e dois de Eimeria e Isospora. Cinco espécies de Eimeria e uma de Isospora foram identificadas: E. scabra $(31,9 \%)$, E. deblieck $(31,9 \%), E$. perminuta $(23,4 \%)$, E. cerdonis $(17,0 \%), E$. scrofae $(12,8 \%)$ e I. suis $(12,8 \%)$.

Os coccídios foram freqüentes na população estudada, especialmente nos jovens. São protozoários comuns em suínos e javalis de todas as idades, predominando em animais jovens. Os adultos, geralmente, atuam como fonte de infecção para os novos. A coccidiose em suínos domésticos geralmente é um problema associado ao confinamento, mas pouco se conhece sobre a prevalência em suídeos silvestres, especialmente em javalis. As espécies identificadas neste estudo foram observadas em suídeos silvestres (BalickaRamisz et al., 2000) e domésticos (Lindsay et al., 1984).

Considerando o número elevado de ovos, larvas, cistos e oocistos de parasitas das diferentes espécies detectadas nas amostras de fezes, conclui-se que os animais do plantel estão altamente infectados, provavelmente devido ao manejo a que são submetidos, o que facilita a disseminação e contaminação das parasitoses.

Palavras-chave: javali, Sus scrofa scrofa, helmintos, protozoários, coccídios

\begin{abstract}
Faecal samples from 79 wild boars (Sus scrofa scrofa) were examined by sedimentation method, zinc sulfate flotation method, and centrifugal flotation in sugar solution for endoparasites research. The results showed that $97.5 \%$ of the samples were positive for helminths and/or protozoa as follows: strongilides (70.9\%), Ascaris suum (46.9\%), Trichuris suis (29.1\%), Metastrongylus sp. (12.6\%), Strongyloides ransomi (3.8\%), Balantidium coli (38.0\%), Entamoeba spp. (15.2\%), Giardia spp. (1.3\%), Blastocystis sp. (12.6\%). The frequency of Entamoeba was higher in young animals. Coccidian oocysts were observed in $59.5 \%$ samples and five species of Eimeria and one Isospora were recovered: Eimeria scabra (31.9\%), E. deblieck (31.9\%), E. perminuta (23.4\%), E. cerdonis (17.0\%), E. scrofae (12.8\%) and Isospora suis $(12.8 \%)$.
\end{abstract}

Keywords: wild boar, Sus scrofa scrofa, helminths, protozoan, coccidian

\section{REFERÊNCIAS BIBLIOGRÁFICAS}

ANTUNES, R. Comida de gaulês? Suin. Industr., v.151, p.24-27, 2001.

ATWILL, E.R.; SWEJTZER, R.A.; PEREIRA, M.D.G. et al. Prevalence of and associated risk factors for shedding Cryptosporidium parvum oocysts and Giardia cysts within feral pig populations in California. Appl. Envir. Microbiol., v.63, p.3946-3949, 1997.

BALICKA-RAMISZ, A.; RAMISZ, A.; PILARCZYK, B. et al. Protozoa from Eimeria genus in wild animals in Poland. Med. Wet., v.56, p.723-724, 2000.

BELOVA, L.M. Fauna of Blastocystis. Parasitologiya, v.29, p.208-213, 1995.

BOREHAM, P.F.L.; STENZEL, D.J. Blastocystis in humans and animals: morphology, biology, and epizootiology. $A d v$. Parasitol., v.32, p.1-70, 1993.

ESLAMI, A.; FARSAD-HAMDI, S. Helminth parasites of wild boar, Sus scrofa, in Iran. $J$. Wildl. Dis., v.28, p.316-318, 1992.

FAUST, E.C.; SAWITZ, W.; TOBIE, J. et al. Comparative efficiency of various technics for the diagnosis of protozoa and helminths in feces. J. Parasitol., v.25, p.241-262, 1939.

GOMES, R.A.; NASCIMENTO, A.A.; BONUTI, M.R. et al. Levantamento helmintológico em javalis (Sus scrofa scrofa) criados em cativeiro no Estado de São Paulo, Brasil. In: CONGRESSO BRASILEIRO DE MEDICINA VETERINÁRIA, 29., 2002, Gramado. Anais... Gramado, 2002. Resumo. CDRoom. 
HUMBERT, J.F.; HENRY, C. Studies on the prevalence and the transmission of lung and stomach nematodes of the Wild Boar (Sus scrofa) in France. J. Wildl. Dis., v.25, p.335-341, 1989.

LINDSAY, D.S.; ERNST, J.V.; CURRENT, W.L. et al. Prevalence of oocysts of Isospora suis and Eimeria spp. from sows on farms with and without a history of neonatal coccidiasis. $J$. Am. Vet. Med. Assoc., v.185, p.419-421, 1984.

LUTZ, A. O. Schistosomum mansoni e a schistosomose, segundo observações feitas no Brasil. Mem. Inst. Oswaldo Cruz, v.11, p.121$155,1919$.

MULLER, G.; SANTOS, T.R.B.; TAVARES, A.J.S. et al. Ocorrência de Metastrongylus apri em javali (Sus scrofa) no município de Pelotas, Rio Grande do Sul, Brasil. In: CONGRESSO LATINOAMERICANO DE PARASITOLOGIA, 15., 2001, São Paulo. J. Braz. Patol., v.39, p.225, 2001.
NAKAUCHI, K. The prevalence of Balantidium coli infection in fifty-six mammalian species. $J$. Vet. Med. Sci., v.61, p.63-65, 1999.

PAKANDL, M. The prevalence of intestinal protozoa in wild and domestic pigs. Vet. Med.(Praha), v.39, p.377-380, 1994.

RODRIGUEZ, J.R.; HERRERA, J.L. Revision de los generos: Eimeria e Isospora (ProtozoaEimeridae) del Sus scrofa domestica (L). Descripciones de las Eimeria guevarai e Isospora neyrai ns spp. Rev. Iber. Parasitol., v.31, p.1-13, 1971.

SHEATHER, A.L. The detection of intestinal protozoa and mange parasites by a flotation technique. J. Comp. Ther., v.36, p.266-275, 1923.

TRIOLA, M. F. Introdução à estatística. 7.ed. Rio de Janeiro: LTC, 1999. 